\title{
A New Custodian for a Realistic Higgsless Model
}

\author{
Giacomo Cacciapaglia $^{a}$, Csaba Csáki ${ }^{a}$, Guido Marandella ${ }^{b}$, and John Terning ${ }^{b}$ \\ ${ }^{a}$ Institute for High Energy Phenomenology \\ Newman Laboratory of Elementary Particle Physics \\ Cornell University, Ithaca, NY 14853, USA \\ ${ }^{b}$ Department of Physics, University of California, Davis, CA 95616. \\ cacciapa@mail.lns.cornell.edu, csaki@mail.lns.cornell.edu, \\ maran@physics.ucdavis.edu, terning@physics.ucdavis.edu
}

\begin{abstract}
We present an example of a realistic Higgsless model that makes use of alternative $S U(2)_{R}$ assignments for the top and bottom quarks recently proposed by Agashe et al. which results in an enhanced custodial symmetry. Using these new representations reduces the deviations in the $Z b_{\ell} \bar{b}_{\ell}$ coupling to $\sim 4 \%$ for a wide range of parameters, while this remaining correction can also be eliminated by varying the localization parameter (bulk mass) for $b_{r}$.
\end{abstract}




\section{Introduction}

It has been realized in the last few years that extra dimensions allow for an alternative approach to electroweak symmetry breaking where the Higgs decouples from the theory [1-3]. With the Higgs localized on a brane one can take the limit $\langle H\rangle \rightarrow \infty$ while the $W$ and $Z$ masses remain finite. In this Higgsless limit the gauge boson masses are set by the size of the extra dimension via Dirichlet boundary conditions (BCs) and $W W$ scattering is unitarized by $W$ and $Z$ Kaluza-Klein $(\mathrm{KK})$ modes $[1,2,4,5]$. If the extra dimension is warped then a bulk gauge symmetry $S U(2)_{L} \times S U(2)_{R} \times U(1)_{X}$ acts $[2,6]$ as a custodial symmetry to ensure the correct ratio of $M_{W} / M_{Z}$ (i.e. a small $T$ parameter [7-10]) at tree level. If the fermions are spread uniformly through the bulk $[11,12]$ then fermion currents are approximately orthogonal to the gauge KK modes so their couplings are suppressed and the $S$ parameter is small ${ }^{1}$. The remaining problem in this class of theories is how to get a large enough top quark mass without messing up the $Z b_{\ell} \bar{b}_{\ell}$ coupling [11]. This final problem can be solved using alternative $S U(2)_{R}$ assignments for the top and bottom quarks that were recently suggested by Agashe et. al. [16]. This alternative is suggested by a combination of custodial symmetry and a $L \leftrightarrow R$ parity symmetry that protects the $Z b_{\ell} \bar{b}_{\ell}$ coupling. This enhanced custodial symmetry suppresses corrections to the $Z b_{\ell} \bar{b}_{\ell}$ vertex, but in Higgsless models it is not sufficient by itself to reach agreement with the experimental bounds. A cancelation, therefore, is still necessary: we identify the simplest scenario where such a cancelation is possible. In this scheme the left-handed (LH) top and bottom quarks are part of a bidoublet of $S U(2)_{L} \times S U(2)_{R}$, while the right-handed $(\mathrm{RH})$ top is a singlet and the $\mathrm{RH}$ bottom is part of an $S U(2)_{R}$ triplet. For a wide range of parameters the deviation of the $Z b_{\ell} \bar{b}_{\ell}$ coupling is reduced to $\sim 4 \%$, and this deviation can be eliminated by varying the bulk mass (localization parameter) of the $b_{r}$.

\section{Fermion masses in Higgsless models}

Higgsless models achieve electroweak symmetry breaking through Dirichlet BCs for gauge fields in an extra dimension $[1,2,9,10]$. The correct ratio of $W$ and $Z$ masses is predicted if the model has a custodial symmetry $[2,6]$. In the standard model (SM) the Higgs sector has an $S U(2)_{L} \times S U(2)_{R}$ symmetry which is broken down to a diagonal $S U(2)_{D}$ custodial symmetry by the Higgs VEV. $S U(2)_{L}$ is a gauge symmetry in the SM, while $S U(2)_{R}$ is a global symmetry that is broken by Yukawa couplings and the hypercharge gauge coupling. (Yukawa couplings would not break the custodial symmetry if the RH fermions were doublets of $S U(2)_{R}$, but then the $t$ and $b$ would have to be degenerate.) In Randall-Sundrum-type models [18], where the SM is embedded in a 5D anti-de Sitter (AdS) space, a custodial symmetry can be achieved by incorporating a bulk gauge symmetry $S U(2)_{L} \times S U(2)_{R} \times U(1)_{X}$ where $S U(2)_{R} \times U(1)_{X}$ is broken down to hypercharge $U(1)_{Y}$ by Dirichlet BCs on the UV brane. This is in accord with the AdS/CFT correspondence, which requires that a global

\footnotetext{
${ }^{1}$ For another suggestion for lowering the $S$ parameter using the condensates of a conformal field theory (CFT) see [14].
} 
symmetry of the strongly coupled CFT corresponds to a gauge symmetry in AdS. On the IR brane, $S U(2)_{L} \times S U(2)_{R}$ is broken down to an $S U(2)_{D}$ custodial symmetry by Dirichlet BCs, analogously to what happens in the SM.

Fermion masses can be easily generated in Higgsless models via Dirac masses ${ }^{2}$ on the IR brane $[10,19]$. In 5D, fermions are vector-like so that each bulk fermion field contains both LH and RH components:

$$
\Psi=\left(\begin{array}{l}
\chi \\
\psi
\end{array}\right)
$$

Here, and in the remainder of the paper, we use $\chi$ to denote the LH fermion and $\psi$ to denote the RH fermion. We will often use subscripts $\ell$ and $r$ to denote LH and RH fermion chirality, while $\mathrm{L}$ and $\mathrm{R}$ always indicate the gauge groups $S U(2)_{L} \times S U(2)_{R}$. A chiral spectrum can be obtained by assigning different BCs to the two components of a 5D fermion: for example, assigning Dirichlet BC's to the RH component $\psi$ is enough to determine the solutions of the bulk equations of motion, and allows for a LH zero mode in $\chi$. One massless flavor thus requires two bulk fermion fields, one with a $\mathrm{LH}$ zero mode and one with a $\mathrm{RH}$ zero mode. The LH and RH zero modes of two fields can be removed by adding a brane Dirac mass for the two fields; this results in new BC's [19] which generate a $z$-dependence incompatible with a zero mode.

The simplest possibility, adopted in Refs. [2,7,11], is to embed the LH and RH SM fermions into $S U(2)_{L}$ and $S U(2)_{R}$ bulk doublets: for instance, for the third generation quarks, $\Psi_{L}=\left(t_{L}, b_{L}\right), \Psi_{R}=\left(t_{R}, b_{R}\right)$ transforming as

$$
(\mathbf{2}, \mathbf{1})_{1 / 6}, \quad(\mathbf{1}, \mathbf{2})_{1 / 6}
$$

of $S U(2)_{L} \times S U(2)_{R} \times U(1)_{X}$. For these representations, the $X$-charge can be identified as $X=(B-L) / 2$. The SM zero modes can be reproduced by the assignment of the following BC's:

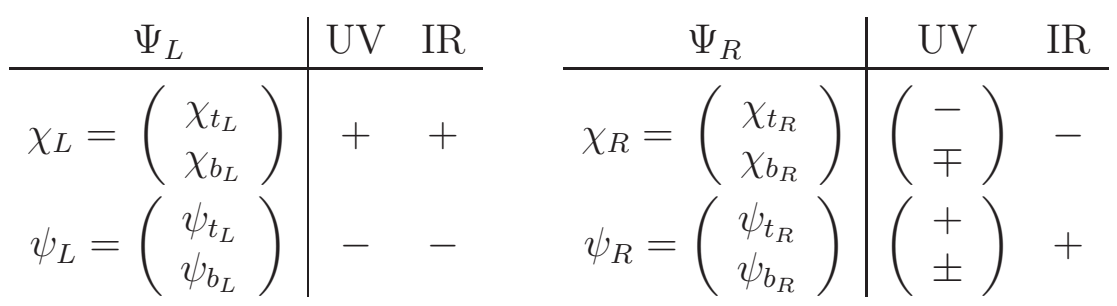

where + stands for a Neumann BC and - stands for a Dirichlet BC, and (in the absence of boundary mass terms) the $\mathrm{LH}$ and $\mathrm{RH}$ components of a field must always have opposite BCs. Note that $S U(2)_{R}$ is broken on the UV brane, so that we can assign different BC's to fields in the $S U(2)_{R}$ doublet: this is the origin of the two possible choices for $\Psi_{R}$. The difference in the two cases is the presence, or not, of a zero mode for the $b_{r}$ : in the latter

\footnotetext{
${ }^{2}$ Note that this scenario can also be generated by a finite Higgs VEV and a localized Higgs Yukawa coupling.
} 
case one can add another $S U(2)_{R}$ doublet $\Psi_{R}^{\prime}$ flipping the BC's between top and bottom quarks so as to get a $\mathrm{RH} b$ zero mode.

A localized mass term on the IR brane of the form:

$$
M\left(\chi_{L} \psi_{R}+\chi_{R} \psi_{L}\right)+\text { h.c. }
$$

replaces [19] the Dirichlet BC's on the IR brane $\left(\psi_{L}=0\right.$ and $\left.\chi_{R}=0\right)$ with:

$$
\psi_{L}=M R^{\prime} \psi_{R}, \quad \chi_{R}=-M R^{\prime} \chi_{L}
$$

It is clear from these BC's that it is not possible to obtain an arbitrarily large top quark mass by increasing $M$. The reason is that in the large $M$ limit the BC's are equivalent to:

$$
\chi_{L}=0, \quad \psi_{R}=0
$$

which just amounts to flipping the BC's on the IR brane, so that the top quark corresponds to the first KK mode of a field with $(+,-)$ BC's whose mass is set by the radius of the extra dimension (rather than the localized Dirac mass).

Note that in order to preserve custodial symmetry, the mass term (2.4) couples to both top and bottom quarks. If the RH $b$ lives in $\Psi_{R}$, the bottom mass can be suppressed with respect to the top mass by a large kinetic term on the UV brane for $\psi_{b_{R}}$. In the case of different UV BCs for $\psi_{t_{R}}$ and $\psi_{b_{R}}$, the mass term (2.4) will not generate the bottom mass, due to the absence of a zero mode for $\psi_{b_{R}}$.

Despite the seeming ease of introducing fermion masses, they in fact pose the main challenge to Higgsless models. First of all, the necessity of having the light fermions spread in the bulk, due to electroweak precision corrections, is potentially dangerous from the point of view of flavor physics [11] without some additional flavor symmetry. The second, more difficult challenge comes from the third generation of quarks: there is a tension between having a heavy top quark and small corrections to the couplings of the LH $b$ with the $Z$ boson. Schematically the large corrections to $Z b_{\ell} \bar{b}_{\ell}$ have two origins. First of all, in order to enhance the $t$ mass, one has to localize the $t$ as close as possible to the IR brane. However, electroweak symmetry breaking is also localized there which distorts the wave functions of the $W$ and $Z$, thus modifying the coupling of any field localized nearby. The second source of deviations is the presence of a massive LH $b^{\prime}$ quark (electric charge $-1 / 3$ ) in the $S U(2)_{R}$ doublet that contains the RH $t$ : this fermion mixes with the $b$ via the IR Dirac mass responsible for the $t$ mass, however it has hypercharge $-1 / 3$ instead of the usual $+1 / 6$. This mixing is a direct consequence of the custodial symmetry. Unfortunately, if the $t$ is not localized extremely close to the IR brane, the heaviness of the $t$ requires the Dirac mass term (2.4) on the IR brane to be very large, so that a large mixing is generated. These two sources together generate large deviations for the entire parameter space.

One possible solution to this problem would be to increase the mass scale on the IR brane, without conflicting with the unitarity bound in the gauge sector. In Ref. [15] this idea was realized in a model with two Randall-Sundrum throats where light fermions and the third generation separately couple to symmetry breaking on two different IR branes. However, in 
order for the gauge sector to be not sensitive to the large scale of the top-sector IR-brane, the top is necessarily strongly coupled to a Higgs and/or resonances living on the top-brane. Although the gauge sector is screened by a weak loop from those strong coupling effects, the top sector is not calculable. ${ }^{3}$

Here we will pursue another possibility that allows for a perturbative top sector: an alternative realization of custodial symmetry [16] that does not require the presence of a $b$-like particle in the bulk field containing the RH $t$. Furthermore, $b_{\ell}$ couples to the diagonal combination of $S U(2)_{L} \times S U(2)_{R}$, that is flat near the IR brane. In this way both of the sources for the deviation of the $Z b_{\ell} \bar{b}_{\ell}$ coupling are suppressed.

\section{An alternative realization of custodial symmetry}

In [16], the authors identified representations of $S U(2)_{L} \times S U(2)_{R}$ that have an enhanced custodial symmetry which can protect the coupling of the $Z$ to a given fermion $\psi$ from nonuniversal corrections. Let us briefly summarize the argument of [16]. One assumes a beyond the SM sector with an $O(4) \sim S U(2)_{L} \times S U(2)_{R} \times P_{L R}$ symmetry, where $P_{L R}$ is the discrete parity interchanging the two $S U(2)$ 's. $O(4)$ is then broken down to $O(3) \sim S U(2)_{D} \times P_{L R}$. If $\psi$ is a +1 eigenstate of $P_{L R}$ then

$$
T_{L}=T_{R}, \quad T_{R}^{3}=T_{L}^{3}
$$

and since the $Q_{L+R}$ charge is protected by $S U(2)_{D}$ (so it is not renormalized) and shifts in $Q_{L}$ and $Q_{R}$ must be equal we have

$$
\delta Q_{L}+\delta Q_{R}=0, \quad \delta Q_{L}=\delta Q_{R}
$$

so the charges are individually protected $\left(\delta Q_{L}=0\right)$, and the $W_{L}^{3}$ coupling is unchanged. In addition fermions with

$$
T_{L}^{3}=T_{R}^{3}=0
$$

cannot couple to $W_{L}^{3}$ at all.

The field we are interested in is the most precisely measured of the third generation, i.e. the LH $b$ quark. We need the field containing $b_{\ell}$ to satisfy (3.7). The minimal choice is to embed $b_{\ell}$ in a bi-doublet of $S U(2)_{L} \times S U(2)_{R}$. Consequently, the RH fermions can be either singlets or triplets of $S U(2)_{L}$ and/or $S U(2)_{R}$. Some possible choices (labeled by $S U(2)_{L} \times S U(2)_{R} \times U(1)_{X}$ quantum numbers) are:

$$
\begin{array}{ll}
(\mathbf{1}, \mathbf{1})_{2 / 3} \supset t_{r} & (\mathbf{1}, \mathbf{3})_{2 / 3} \text { and } / \text { or }(\mathbf{3}, \mathbf{1})_{2 / 3} \supset t_{r}, b_{r} \\
(\mathbf{1}, \mathbf{1})_{-1 / 3} \supset b_{r} & (\mathbf{1}, \mathbf{3})_{-1 / 3} \text { and /or }(\mathbf{3}, \mathbf{1})_{-1 / 3} \supset t_{r}, b_{r} .
\end{array}
$$

\footnotetext{
${ }^{3}$ An analogous separation of scales for fermion and gauge boson masses in the deconstructed version of the model has been proposed in [17].
} 
For instance, the third generation of quarks could be obtained from the following representations:

$$
\begin{aligned}
\Psi_{L} \sim(\mathbf{2}, \mathbf{2})_{2 / 3} & \supset\left(t_{\ell}, b_{\ell}\right) \\
t_{R} \sim(\mathbf{1}, \mathbf{1})_{2 / 3} & \supset t_{r} \\
\Psi_{R} \sim(\mathbf{1}, \mathbf{3})_{2 / 3} & \supset b_{r}
\end{aligned}
$$

This is one of the two cases where the realization of the custodial symmetry identified in [16] protects the $Z b_{\ell} \bar{b}_{\ell}$ couplings ${ }^{4}$. Note that the field containing $t_{r}$ does not contain any field that can mix with $b_{\ell}$ : in other words, the $t$ mass will not induce any mixing in the $b$ sector. The $b$ mass is generated by a different (and smaller) Dirac mass.

Notice that in order for the enhanced custodial symmetry to protect the $b, \Psi_{R}$ should be completed to a full $O(4)$ representation: $\Psi_{R}=(\mathbf{3}, \mathbf{1})_{2 / 3} \oplus(\mathbf{1}, \mathbf{3})_{2 / 3}$. This is because in the $S U(2)_{R}$ triplet $(\mathbf{1}, \mathbf{3})_{2 / 3}$, the component with $T_{R}^{3}=-1$ has the quantum numbers of a $b$. We can choose the BC's for this field such that a zero mode is only present in the RH component, while the LH modes are all massive. However, the Dirac mass needed to get the $b$ mass mixes it with the LH component living in $\Psi_{L}$. Thus, the LH $b$ lives partly in $(\mathbf{1}, \mathbf{3})_{2 / 3}$. In order to protect the $Z b_{\ell} \bar{b}_{\ell}$ vertex, we need to complete the representation with a $S U(2)_{L}$ triplet: its component with $T_{L}^{3}=-1$ will also mix with $b_{L}$, and it will cancel out the contribution of the $S U(2)_{R}$ triplet (component with $T_{R}^{3}=-1$ ) to $Z b_{\ell} \bar{b}_{\ell}$. Usually, since the $b$ quark mass is small, it can be neglected and completing the RH fields to full $O(4)$ representations is irrelevant. However, in the context of the Higgsless model, due to possible different localizations of $\Psi_{L}$ and $\Psi_{R}$ the Dirac mass needed to get the $b$ mass can be quite large. Thus it might seem that completing $\Psi_{R}$ to a full $O(4)$ representations could be crucial. We will come back to this point in the next section.

For the time being let us consider $\Psi_{R}$ to be a $S U(2)_{R}$ triplet and write, in $5 \mathrm{D}$ components,

$$
\Psi_{L}=\left(\begin{array}{cc}
q_{L} & Q_{L}
\end{array}\right)=\left(\begin{array}{cc}
t_{L} & X_{L} \\
b_{L} & T_{L}
\end{array}\right), \quad \Psi_{R}=\left(\begin{array}{c}
X_{R} \\
T_{R} \\
b_{R}
\end{array}\right), \quad t_{R},
$$

where all these fermion fields are bulk fields. For example, $t_{R}$ contains both $\mathrm{LH}$ and $\mathrm{RH}$ components $\chi_{t_{R}}$ and $\psi_{t_{R}}$. Notice also that the charges of the extra fields are $Q[T]=2 / 3$ and $Q[X]=5 / 3$, so that $T$ will mix with $t$. The quantum numbers, and BC's, of the various fields are shown in Tables 1 and 2. As usual, $Y=T_{R}^{3}+X, Q=T_{L}^{3}+Y$, and the BC's ensure that the only zero modes correspond to SM fields.

The mass terms can be written as:

$$
\mathcal{L}_{I R}=M_{3}\left[\frac{1}{\sqrt{2}} T_{R}\left(t_{L}+T_{L}\right)+b_{R} b_{L}+X_{R} X_{L}\right]+\frac{M_{1}}{\sqrt{2}} t_{R}\left(t_{L}-T_{L}\right)+\text { h.c. }
$$

Under the unbroken $S U(2)_{D}$ on the IR brane, $\left(t_{L}-T_{L}\right) / \sqrt{2}$ is the singlet component of $\Psi_{L}$, while $\left(X_{L},\left(t_{L}+T_{L}\right) / \sqrt{2}, b_{L}\right)$ is the triplet. Note that the $t_{L}$ is mixed with the massive

\footnotetext{
${ }^{4}$ In the other case, $t_{r}$ is embedded in a $(\mathbf{1}, \mathbf{3})_{2 / 3} \oplus(\mathbf{3}, \mathbf{1})_{2 / 3}$. We focus on the former scenario because it involves fewer bulk fields: however, all of the following discussion can be applied to the latter case as well.
} 


\begin{tabular}{c|rr} 
& $T_{L}^{3}$ & $T_{R}^{3}$ \\
\hline$X_{L}$ & $1 / 2$ & $1 / 2$ \\
$T_{L}$ & $-1 / 2$ & $1 / 2$ \\
$t_{L}$ & $1 / 2$ & $-1 / 2$ \\
$b_{L}$ & $-1 / 2$ & $-1 / 2$ \\
$X_{R}$ & 0 & 1 \\
$T_{R}$ & 0 & 0 \\
$b_{R}$ & 0 & -1 \\
$t_{R}$ & 0 & 0
\end{tabular}

\begin{tabular}{c|cc} 
& $\mathrm{UV}$ & $\mathrm{IR}$ \\
\hline$\chi_{q_{L}}$ & + & + \\
$\chi_{Q_{L}}$ & - & + \\
$\psi_{X_{R}}$ & - & + \\
$\psi_{T_{R}}$ & - & + \\
$\psi_{b_{R}}$ & + & + \\
$\psi_{t_{R}}$ & + & +
\end{tabular}

Table 1: Quantum numbers of the bulk fields, and Table 2: BC's of the bulk fields.

$T_{L}$, so that a larger Dirac mass is required than was the case for the representations (2.2). This implies that the $t$ has to be localized closer to the IR brane than in the previous case. Moreover, the $b$ only feels the smaller $M_{3}$ term, so the $b$ wave function is not distorted as much as the $t$.

\section{Results for Higgsless model}

We first consider applying the new representation (3.11) to the light fermions, whose masses are so small that mass dependent corrections can be neglected. In Fig. 1 we show the coupling of the LH down-type quark $d_{\ell}$ with the $Z$ with respect to the SM coupling: for the alternative representation (3.11), the $Z d_{\ell} \bar{d}_{\ell}$ coupling deviates by $+4 \%$ to $+5 \%$, independently of the bulk mass of the $\Psi_{L}$ fields. This correction has a universal origin which is equivalent to a large $S$ parameter [11,20]. For light fermions in the representations (2.2), this can be compensated by a suitable value of the bulk mass ${ }^{5} c_{L} \simeq 0.45$, as in [11]. With the alternative representation it is not possible to cancel the correction, since the coupling is now protected by the enhanced symmetry and does not depend on the localization of the fermions. This fact implies that the alternative representation (3.11) cannot be used for the light fermions in Higgsless models due to this large vertex correction. There is a very clear way of understanding this in terms of KK modes with electroweak symmetry breaking treated as a perturbation: the correction is generated by a tree level graph where the $b_{\ell}$ emits a KK mode of the $Z$, that mixes with the zero mode due to electroweak symmetry breaking BC's on the IR brane. There are three different kind of gauge KK modes: those from $S U(2)_{L}$, those from the combination of $S U(2)_{R}$ and $U(1)_{X}$ that is unbroken on the UV brane (which couples through hypercharge $Y$ ), and those from the broken combination. In the limit of flat fermions $\left(c_{L}=0.5\right)$, only the latter $\mathrm{KK}$ modes will contribute and give the few $\%$ deviation we observe, because they do not have a zero mode and are thus not orthogonal to the flat wave function. When $c_{L} \neq 0.5$, the other modes will also contribute, but, due to the enhanced symmetry, they will cancel so as to keep the deviation constant.

\footnotetext{
${ }^{5}$ In units of the AdS scale $1 / R$.
} 

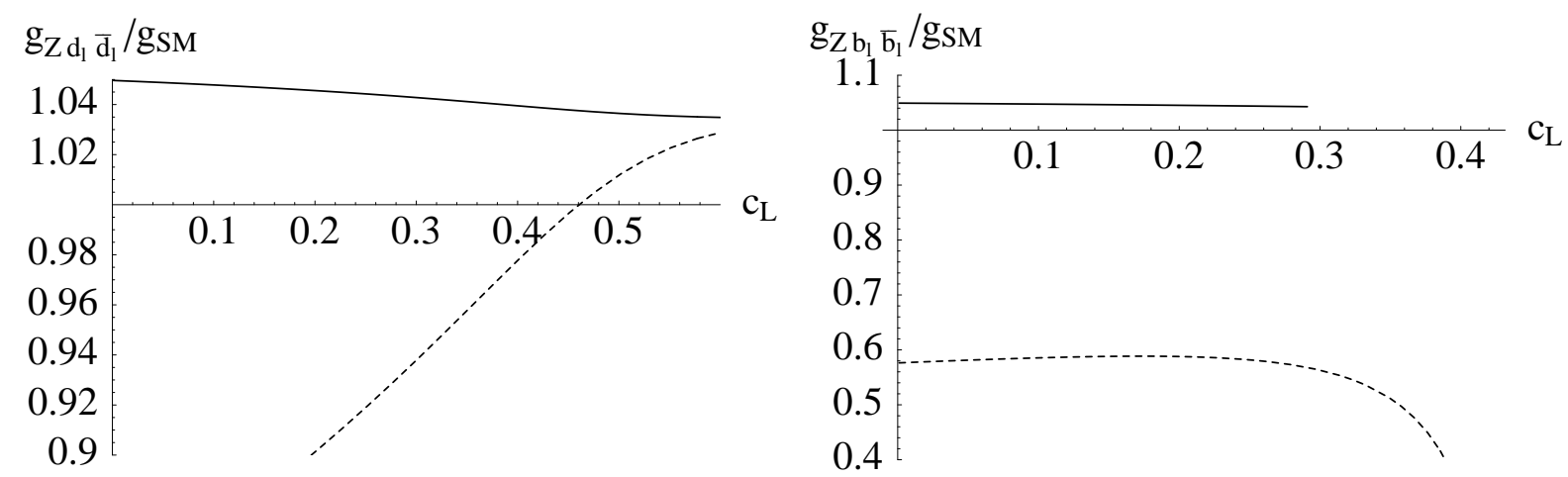

Figure 1: Deviations in the coupling of the down-type quarks as a function of the bulk mass $c_{L}$ using the alternative realization of custodial symmetry, where $\Psi_{L}=(\mathbf{2}, \mathbf{2}), \Psi_{R}=$ $(\mathbf{1}, \mathbf{3}), t_{R}=(\mathbf{1}, \mathbf{1})$ (continuous line) and the original case, where $\Psi_{L}=(\mathbf{2}, \mathbf{1}), \Psi_{R}=(\mathbf{1}, \mathbf{2})$ (dashed line). In the left panel, we consider a light quark (first and second generation). In the right panel, we show the $b$ quark, with $c_{R}^{t}=0$, while neglecting the $b$ mass.

On the other hand, in the original case (2.2), we can tune the parameters such that the contributions completely cancel each other.

Next, we neglect the $b$ mass $\left(M_{3}=0\right)$, and consider only the $t$ mass. As already mentioned, despite the fact that $\Psi_{R}$ should be completed to a full $O(4)$ representation, if only $M_{1}$ is considered $Z b_{\ell} \bar{b}_{\ell}$ remains protected: the $t$ mass does not affect the coupling of the $b$, and the correction remains at +4 to $+5 \%$. In the old case (2.2), the corrections were typically $\sim-40 \%$, due to the large mixing of the $\chi_{b_{L}}$ with a LH field contained in the $\Psi_{R}$ doublet: thus, the new symmetry allows for a significant reduction in the correction, but it is still not enough to be compatible with the experimental bounds. Another difference between the two cases is that with the new representation (3.11) the approximate zero modes in $\Psi_{L}$ have to be more localized toward the IR brane in order to produce the observed $t$ mass: in fact, $c_{L} \lesssim 0.3$ is required, compared with $c_{L} \lesssim 0.45$ in the old case. As we mentioned before the reason behind this is the mixing of the $t$ and $T$ fields. The results are shown in Fig. 1.

Next we analyze the same corrections, taking into account the $b$ mass. As we said in Sec. 3 if $M_{3}$ is considered, and $\Psi_{R}$ is an $S U(2)_{R}$ triplet, neither (3.7) nor (3.9) are satisfied. So, we expect deviations in the $Z b_{\ell} \bar{b}_{\ell}$ coupling. Notice that if we completed $\Psi_{R}=(\mathbf{3}, \mathbf{1})_{2 / 3} \oplus(\mathbf{1}, \mathbf{3})_{2 / 3}$ nothing would change with respect to the case with $M_{3}=0$ : the deviation would remain between 4 and $5 \%$ since the enhanced custodial symmetry would be at work. If a sizeable Dirac mass is necessary to fit the $b$ mass, then the LH mode in $b_{R}$ (the triplet component with electric charge $-1 / 3$ but with hypercharge $2 / 3$ ) mixes with the LH mode in $b_{L}$ and can cancel the correction to $Z b_{\ell} \bar{b}_{\ell}$. The size of the Dirac mass needed to get the $b$ mass crucially depends on the bulk mass parameter $c_{R}^{b}$ for the field $\Psi_{R}$. If this is large and negative, i.e. the approximate zero mode in $\Psi_{R}$ is localized near the UV brane, the overlap between $b_{L}$ and $b_{R}$ is small, and the Dirac mass is of order the IR scale $1 / R^{\prime}$. Such a big Dirac mass induces a sizeable mixing between the LH modes living in $b_{L}$ and $b_{R}$, giving a negative correction to the $Z b_{\ell} \bar{b}_{\ell}$ coupling. Note that the sign of this correction goes in the right direction to agree 


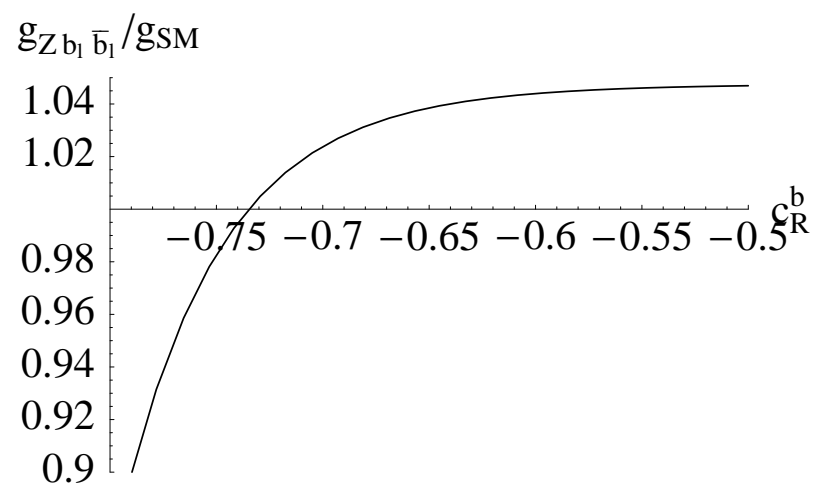

Figure 2: Deviations in the coupling $Z f_{\ell} \bar{f}_{\ell}$ as a function of the bulk mass $c_{R}^{b}$ of $S U(2)_{R}$ triplet containing the $b_{R}$ using the alternative realization of custodial symmetry. The other bulk masses have been set to $c_{L}=0.1, c_{R}^{t}=0$.

with the SM. In Fig. 2 we plot the correction to the $Z b_{\ell} \bar{b}_{\ell}$ coupling as a function of $c_{R}^{b}$. In this plot, we choose $c_{L}=0.1$ while the bulk mass parameter corresponding to $t_{R}$ has been set to $c_{R}^{t}=0$. In the notation of ref. [2], we have set the AdS scale to $1 / R=10^{8} \mathrm{GeV}$, while the IR scale is determined by $M_{Z}, G_{F}$ and $\alpha\left(M_{Z}\right)$ to be $1 / R^{\prime}=282 \mathrm{GeV}$. This plot has a mild dependence on $c_{L}$ and $c_{R}^{t}$. For $c_{R}^{b} \sim-0.75$, the two contributions cancel out, and the coupling agrees with the SM to the required accuracy. To summarize, in the scenario where the approximate zero modes of $t_{L}, t_{R}, b_{L}$ are localized near the IR brane $\left(c_{L} \lesssim 0.3, c_{R}^{t} \simeq 0\right)$, and $b_{R}$ is localized close to the UV brane $\left(c_{R}^{b} \simeq-0.75\right)$, allows for a fully realistic Higgsless model: the $t$ mass can be obtained, while corrections to the $Z b_{\ell} \bar{b}_{\ell}$ coupling can be made arbitrarily small.

We can now examine the couplings of the third generation quarks and gauge bosons. Setting $c_{L}=0.1, c_{R}^{t}=0, c_{R}^{b}=-0.73$, we find the values shown in Table 3 .

\begin{tabular}{c|l} 
& frac. of SM \\
\hline \hline$Z b_{\ell} \bar{b}_{\ell}$ & 1.004 \\
$Z b_{r} \bar{b}_{r}$ & 0.993 \\
$Z t_{\ell} \bar{t}_{\ell}$ & 0.461 \\
$Z t_{r} \bar{t}_{r}$ & 1.908 \\
$W t_{\ell} \bar{b}_{\ell}$ & 0.862 \\
$W t_{r} \bar{b}_{r}$ & $3 \cdot 10^{-4} g_{W t_{\ell} \bar{b}_{\ell}}$
\end{tabular}

Table 3: The couplings of the $t$ and $b$ to $W$ and $Z$ in a Higgsless model. We have taken the AdS scale to be $1 / R=10^{8} \mathrm{GeV}$, the compactification scale to be $1 / R^{\prime}=282 \mathrm{GeV}$.

Notice that the deviations in the couplings of the $Z$ to the $t$ lead to a modification of the SM one-loop contribution to the $Z b_{\ell} \bar{b}_{\ell}$ coupling. Analogously, choosing for $\Psi_{R}$ to be an 


\begin{tabular}{c|lc|l}
$M_{t^{\prime}}$ & $450 \mathrm{GeV}$ & $g_{Z^{\prime} t_{L} \bar{t}_{L}}$ & $1.83 g_{Z t_{L} \bar{t}_{L}}$ \\
$M_{b^{\prime}}$ & $664 \mathrm{GeV}$ & $g_{Z^{\prime} t_{R} \bar{t}_{R}}$ & $4.02 g_{Z t_{R} \bar{t}_{R}}$ \\
$M_{W^{\prime}}$ & $695 \mathrm{GeV}$ & $g_{Z^{\prime} b_{L} \bar{b}_{L}}$ & $3.77 g_{Z b_{L} \bar{b}_{L}}$ \\
$M_{Z^{\prime}}$ & $690 \mathrm{GeV}$ & $g_{Z^{\prime} b_{R} \bar{b}_{R}}$ & $0.26 g_{Z b_{R} \bar{b}_{R}}$ \\
$M_{Z^{\prime \prime}}$ & $714 \mathrm{GeV}$ & $g_{Z W W}$ & $1.018 g c_{W}$ \\
$M_{G^{\prime}}$ & $714 \mathrm{GeV}$ & $g_{Z Z W W}$ & $1.044 g^{2} c_{W}^{2}$ \\
$g_{W^{\prime} u \bar{d}}$ & $0.07 g$ & $g_{W W W W}$ & $1.032 g^{2}$ \\
$g_{Z^{\prime} q \bar{q}}$ & $0.14 g_{Z q \bar{q}}$ & $g_{Z^{\prime} W W}$ & $0.059 g c_{W}$ \\
$g_{G^{\prime} q \bar{q}}$ & $0.22 g_{c}$ & $g_{Z W^{\prime} W}$ & $0.051 g c_{W}$
\end{tabular}

Table 4: Summary of the realistic Higgless model for the following choice of parameters: $1 / R=10^{8} \mathrm{GeV}, 1 / R^{\prime}=282 \mathrm{GeV}, g_{5}=0.66\left(R \log R^{\prime} / R\right)^{1 / 2}, \tilde{g}_{5}=0.42\left(R \log R^{\prime} / R\right)^{1 / 2}$. For the light fermions $c_{L}=0.46$, while for the third generation $c_{L}=0.1, c_{R}^{t}=0, c_{R}^{b}=-0.73$.

incomplete $O(4)$ representation will produce, through loop effects, a breaking of the $L \leftrightarrow R$ symmetry. This might feed back into corrections to the $Z b_{l} \bar{b}_{l}$ coupling. All these corrections are at most around $1 \%$ and a suitable value of $c_{R}^{b}$ can always compensate it, as it is clear from Fig. 2. On the other hand the deviation of the coupling with the $W$ is small enough not to affect present measurements. The $W t_{\ell} b_{\ell}$ coupling will be measured by single $t$ production, first at the Tevatron and eventually at the LHC with a precision around 5\% [22].

We now summarize the features of a realistic Higgsless model at a fairly typical point in the parameter space. In Table 4 we give the spectrum of the first KK excitations of top and bottom quarks, the gauge bosons, and their couplings to the SM fermions as a fraction of the analogous SM coupling. We have set the AdS scale to be $1 / R=10^{8} \mathrm{GeV}$, the IR scale to be $1 / R^{\prime}=282 \mathrm{GeV}$, and the ratio between the $U(1)$ and the $S U(2) 5 \mathrm{D}$ gauge couplings to be $g_{5}=0.66\left(R \log R^{\prime} / R\right)^{1 / 2}, \tilde{g}_{5}=0.42\left(R \log R^{\prime} / R\right)^{1 / 2}$. The bulk mass for the light fermions is $c_{L}=0.46$, which makes the $S$ parameter vanish. The parameters for the third generation are as described above: $c_{L}=0.1, c_{R}^{t}=0, c_{R}^{b}=-0.73$.

For the chosen value of $R$ the gauge KK modes are close to the Tevatron bounds, and so could conceivably be discovered or ruled out soon. As the Tevatron bounds move up, they effectively raise the bound on $1 / R$, and all the gauge $\mathrm{KK}$ masses move up [11]. If the $W^{\prime}$ and $Z^{\prime} \mathrm{KK}$ masses go above $1 \mathrm{TeV}$, then $W W$ scattering becomes strongly coupled and the Higgsless scenario is no longer calculable $[5,21]$.

It is also interesting to notice the deviations in the 3- and 4-gauge boson couplings: those deviations are one of the main signatures of Higgsless models and do not depend on the details of the fermion sector. A plot of such deviations as a function of the parameters of the model in the gauge sector can be found in [23]. Triple gauge boson couplings could be measured at one part in a thousand at a future ILC. 


\section{Conclusions}

We have found an example of a realistic Higgsless model that makes use of alternative $S U(2)_{R}$ assignments for the top and bottom quarks recently proposed by Agashe et al. [16]. Using these $L \leftrightarrow R$ symmetric representations reduces the deviations in the $Z b_{\ell} \bar{b}_{\ell}$ coupling, even when the large $t$ quark mass is taken into account. If the bottom quark mass is also taken into account, then for a particular value of the $b_{R}$ bulk mass the $Z b_{\ell} \bar{b}_{\ell}$ coupling is in agreement with precision electroweak measurements.

One can take the model described here as an existence proof for the possibility of Higgsless extra-dimensional models actually being realized in our Universe. They also can provide a reasonable benchmark for comparison with experiment. It would still be interesting to find more elegant Higgsless models where the agreement with precision electroweak measurements is guaranteed by additional symmetries rather than parameter adjustment. Nature, however is the ultimate arbiter of what is sufficiently elegant, and hopefully in a few years the LHC will reveal the scenario that Nature has chosen.

\section{Acknowledgements}

We thank Kaustubh Agashe, Roberto Contino, Alex Pomarol, and Marco Serone for useful discussions and comments. C.C. and J.T. thank the Galileo Galilei Institute for Theoretical Physics for their hospitality and the INFN for partial support during the completion of this work. The research of G.C. and C.C. is supported in part by the DOE OJI grant DE-FG0201ER41206 and in part by the NSF grants PHY-0139738 and PHY-0098631. G.M. and J.T. are supported by the US Department of Energy grant DE-FG02-91ER40674.

\section{References}

[1] C. Csáki, C. Grojean, H. Murayama, L. Pilo and J. Terning, Phys. Rev. D 69, 055006 (2004) hep-ph/0305237.

[2] C. Csáki, C. Grojean, L. Pilo and J. Terning, Phys. Rev. Lett. 92, 101802 (2004) hep-ph/0308038.

[3] R. Casalbuoni, S. De Curtis and D. Dominici, Phys. Rev. D 70 (2004) 055010 hep-ph/0405188; R. S. Chivukula, E. H. Simmons, H. J. He, M. Kurachi and M. Tanabashi, Phys. Rev. D 70 (2004) 075008 hep-ph/0406077; H. Georgi, Phys. Rev. D 71, 015016 (2005) hep-ph/0408067; R. Sekhar Chivukula, E. H. Simmons, H. J. He, M. Kurachi and M. Tanabashi, Phys. Rev. D 71 (2005) 035007 hep-ph/0410154 R. S. Chivukula, et. al., hep-ph/0607124.

[4] R. S. Chivukula, D. A. Dicus and H. J. He, Phys. Lett. B 525, 175 (2002) hep-ph/0111016; R. S. Chivukula and H. J. He, Phys. Lett. B 532, 121 (2002) hep-ph/0201164 R. S. Chivukula, D. A. Dicus, H. J. He and S. Nandi, 
hep-ph/0302263; S. De Curtis, D. Dominici and J. R. Pelaez, Phys. Lett. B 554, 164 (2003) hep-ph/0211353 Phys. Rev. D 67, 076010 (2003) hep-ph/0301059; Y. Abe, N. Haba, Y. Higashide, K. Kobayashi and M. Matsunaga, Prog. Theor. Phys. 109, 831 (2003) hep-th/0302115.

[5] M. Papucci, hep-ph/0408058.

[6] K. Agashe, A. Delgado, M. J. May and R. Sundrum, JHEP 0308, 050 (2003) hep-ph/0308036.

[7] G. Cacciapaglia, C. Csáki, C. Grojean and J. Terning, Phys. Rev. D 70, 075014 (2004) hep-ph/0401160.

[8] H. Davoudiasl, J. L. Hewett, B. Lillie and T. G. Rizzo, "Higgsless electroweak symmetry breaking in warped backgrounds: Constraints and signatures," Phys. Rev. D 70, 015006 (2004) arXiv:hep-ph/0312193.

[9] Y. Nomura, JHEP 0311, 050 (2003) hep-ph/0309189.

[10] R. Barbieri, A. Pomarol and R. Rattazzi, Phys. Lett. B 591 (2004) 141 hep-ph/0310285.

[11] G. Cacciapaglia, C. Csáki, C. Grojean and J. Terning, Phys. Rev. D 71, 035015 (2005) hep-ph/0409126.

[12] R. Foadi, S. Gopalakrishna and C. Schmidt, Phys. Lett. B 606, 157 (2005) hep-ph/0409266;

[13] R. S. Chivukula, E. H. Simmons, H. J. He, M. Kurachi and M. Tanabashi, Phys. Rev. D 71, 115001 (2005) hep-ph/0502162; R. Casalbuoni, S. De Curtis, D. Dolce and D. Dominici, Phys. Rev. D 71, 075015 (2005) hep-ph/0502209.

[14] J. Hirn and V. Sanz, hep-ph/0606086

[15] G. Cacciapaglia, C. Csaki, C. Grojean, M. Reece and J. Terning, Phys. Rev. D 72, 095018 (2005) hep-ph/0505001.

[16] K. Agashe, R. Contino, L. Da Rold and A. Pomarol, hep-ph/0605341.

[17] R. Foadi and C. Schmidt, Phys. Rev. D 73, 075011 (2006) hep-ph/0509071

[18] L. Randall and R. Sundrum, Phys. Rev. Lett. 83, 3370 (1999) hep-ph/9905221.

[19] C. Csáki, C. Grojean, J. Hubisz, Y. Shirman and J. Terning, Phys. Rev. D 70, 015012 (2004) hep-ph/0310355.

[20] C. Grojean, W. Skiba and J. Terning, Phys. Rev. D 73, 075008 (2006) hep-ph/0602154

G. Cacciapaglia, C. Csaki, G. Marandella and A. Strumia, hep-ph/0604111. 
[21] J. L. Hewett, B. Lillie and T. G. Rizzo, JHEP 0410 (2004) 014 hep-ph/0407059.

[22] J. Alwall et al., hep-ph/0607115.

[23] G. Cacciapaglia, in "Les Houches 'Physics at TeV colliders 2005' Beyond the standard model working group: Summary report," B. C. Allanach et al., hep-ph/0602198. 